\title{
RENORMALIZED PATH INTEGRAL IN QUANTUM MECHANICS
}

\author{
R.J. HENDERSON and S. G. RAJEEV \\ Henderson or Rajeev@urhep.pas.rochester.edu \\ Department of Physics and Astronomy, University of Rochester, Rochester, NY 14627
}

\begin{abstract}
We obtain direct, finite, descriptions of a renormalized quantum mechanical system with no reference to ultraviolet cutoffs and running coupling constants, in both the Hamiltonian and path integral pictures. The path integral description requires a modification to the Wiener measure on continuous paths that describes an unusual diffusion process wherein colliding particles occasionally stick together for a random interval of time before going their separate ways.
\end{abstract}

\section{Introduction}

The presence of ultraviolet divergences in the quantum field theories of the standard model, and the need for an awkward renormalization procedure to make these theories well-defined, might be viewed as evidence that quantum field theory is not the proper framework for a fundamental theory of elementary particles. Some exotic and finite theory, such as string theory, might be more conceptually accurate and less mathematically cumbersome. On the other hand, a more conservative point of view is that renormalizable interactions can be given a finite description, which avoids renormalization, without the necessity of discarding the framework of quantum field theory.

It would be aesthetically pleasing and probably computationally useful to construct a theory of renormalizable interactions which is finite at the outset and does not require the seemingly artificial limiting procedures of renormalization to be well-defined. Such a description is presently beyond our grasp, but here we construct a finite description of a renormalizable quantum mechanical system that suggests that quantum field theory can possibly accomodate renormalizable interactions through a choice of the Hamiltonian domain rather than the addition of an interaction term in the Lagrangian. In the case we examine, we thus find evidence that we can eliminate the need for renormalization altogether by taking the conservative point of view which requires no exotic replacement for quantum field theory. An analagous finite description of renormalizable interactions in quantum field theory seems, therefore, a worthwhile goal. 


\section{A Renormalizable Quantum Mechanical System}

It has been recognized for some time (see [1], [2], [3], [4]) that renormalizable ultraviolet divergences are not restricted to quantum field theories, but can occur as well even in nonrelativistic quantum mechanics. A scale invariant Hamiltonian that admits a negative energy bound state necessarily obtains a continuum of negative energy states extending down to arbitrarily negative energies such that there is no ground state. The system, without renormalization, is thus unstable, and ill-defined.

The example we treat is representative of this situation. The attractive Dirac delta function potential in two dimensions with nonrelativistic kinetic energy has been treated (see [1], [2], [4]) by the conventional renormalization recipe: regularize with an ultraviolet cutoff, allow the coupling constant to run (depend on the cutoff), and remove the cutoff keeping some physical observable such as the ground-state energy fixed. In this way divergences are removed, and the dimensionless coupling constant characterizing the system is traded for a dimensionful parameter, such as the ground state energy. This prescription may be administered either perturbatively (order by order in the coupling constant) or nonperturbatively, but in either case the philosophy is the same: solve a regularized, non-physical, system first, then take limits of the solutions to obtain physical results.

Whether or not a direct renormalized description of the system to be solved can be given to avoid such limiting procedures and non-physical intermediate results is then a natural question. Here we give such a description of the delta function potential in two dimensions, first in the Hamiltonian and then in the path integral pictures. The result is a better understanding of the role of the domain of the Hamiltonian in the former, and the description of an interesting alternative to the Wiener measure in the latter.

Our starting point could be the Hamiltonian:

$$
H_{g}=-\Delta-g \delta^{2}(\bar{x})
$$

where $\Delta$ is the two dimensional Laplacian, and $g$ is a positive dimensionless number. In momentum space the Schrodinger equation is then:

$$
p^{2} \Psi(\bar{p})-\frac{g}{(2 \pi)^{2}} \int d^{2} p \Psi(\bar{p})=\lambda \Psi(\bar{p})
$$

Due to scale invariance, this equation admits bound states for any energy, $\lambda$, less than zero. They have the simple form which follows from a rearrangement 
of (2):

$$
\Psi(\bar{p})=\frac{\frac{g}{(2 \pi)^{2}} \int d^{2} p \Psi(\bar{p})}{p^{2}+|\lambda|}
$$

However, integrating this equation over momentum space reveals a problem: $\int d^{2} p \Psi(\bar{p})$ is not finite. There is a logarithmic divergence in the integral at high momenta. One way to cure this illness is to regularize by introducing a large momentum cutoff $\Lambda$ and allowing the coupling constant $g$ to depend on $\Lambda$ in the way which keeps the bound state energy constant as $\Lambda$ is removed (taken to infinity). Integrating the Schrodinger equation up to the cutoff $\Lambda$ gives:

$$
1=\frac{g(\Lambda)}{2 \pi} \int_{0}^{\Lambda} \frac{p d p}{p^{2}+\left|\lambda_{0}\right|}
$$

which has the problem that the integral diverges logarithmically if $\Lambda \rightarrow \infty$. The proper dependence of $g$ on $\Lambda$ to keep $\lambda_{0}$ fixed is:

$$
g(\Lambda)=\frac{2 \pi}{\ln \left(\frac{\Lambda^{2}}{\left|\lambda_{0}\right|}+1\right)}
$$

The choice of $\lambda_{0}(<0)$ is arbitrary, but must be made, and picks out just one of the uncountable number of possible bound states to survive the renormalization procedure. With this choice, the cutoff can be removed, and the parameter $g$ disappears from the problem, replaced by the new parameter $\lambda_{0}$, the energy of the single bound state of the system. All physical observables (e.g. scattering amplitudes) may be calculated by solving this system with the cutoff in place, and then taking the limit $\Lambda \rightarrow \infty$ with $g$ replaced by the expression in (5).

Nonperturbative and successful as this method is, we might ask for a direct description in the Hamiltonian picture wherein the cutoff $\Lambda$ need not appear at all. If such a finite description of a renormalizable system is possible, it should be easiest to find it here in our simple case. This direct description may improve our understanding of the role of renormalization and possibly serve as a guide to finding a similar point of view in the more complicated case of quantum field theory.

The system we have described is asymptotically free. This means that the coupling constant, $g$, goes to zero as $\Lambda$ is taken to infinity. Nonetheless, if we take this limit in the momentum space Hamiltonian, with the dependence of $g$ an $\Lambda$ given above, the Hamiltonian is not just $p^{2}$. An interaction term survives, and the Schrodinger equation becomes:

$$
p^{2} \Psi(\bar{p})-\lim _{p \rightarrow \infty} p^{2} \Psi(\bar{p})=\lambda \Psi(\bar{p})
$$


The renormalized Hamiltonian in (6) appears to depend on no parameters. However, for the Hamiltonian to be self-adjoint, its domain must be specified carefully. The domain here is determined by the bound state energy (the parameter of the theory) and consists of wavefunctions satisfying:

$$
\int d^{2} p\left(\Psi(\bar{p})-\frac{\eta_{\Psi}}{p^{2}+\mu^{2}}\right)=0
$$

where $\lambda_{0}=-\mu^{2}$ and $\eta_{\Psi} \equiv \lim _{p \rightarrow \infty} p^{2} \Psi(\bar{p})$.

In real space, this equation picks out wavefunctions that diverge logarithmically at the origin, but are still square-integrable. This Hamiltonian operator is the momentum space equivalent of the self-adjoint extension of the two-dimensional Laplacian, which is described in [5]. The parameter $\mu^{2}$ can be any positive real number, each value corresponding to a different self-adjoint operator. It is related to the parameter $\alpha$ of [5] by:

$$
\ln \left(2 / \mu^{2}\right)=2 \gamma+4 \pi \alpha
$$

where $\gamma$ is Euler's constant. The bound state wavefunction is:

$$
\Psi_{\lambda_{0}}(\bar{p})=\frac{1}{p^{2}+\mu^{2}}
$$

and the zero angular momentum scattering states with energy $\lambda$ are:

$$
\Psi_{\lambda}(\bar{p})=\frac{1}{k} \ln \left(\frac{\mu^{2}}{k^{2}}\right) \delta(p-k)+\frac{2}{k^{2}-p^{2}}
$$

The non-zero angular momentum scattering states are simply the free ones: plane waves in real space, delta functions in momentum space.

Thus we conclude that this asymptotically free interaction in quantum mechanics, corresponds in momentum space to the unusual Hamiltonian in (6) and in configuration space to the free Hamiltonian with a boundary condition requiring angular momentum zero wavefunctions to diverge at the origin.

That an asymptotically free renormalizable interaction can be specified directly in terms of the domain of the Hamiltonian in configuration space has been discovered previously, in the context of the large- $\mathrm{N}$ limit of the 1+1dimensional massless non-abelian Thirring (or Gross-Neveu) model, [6]. 


\section{The Feynman-Kac Formula and Path Integral Picture}

Generally, quantum mechanical probability amplitudes and expectation values may be calculated in Euclidean time using the Feynman-Kac formula:

$$
<\bar{x}_{1}\left|e^{-t H}\right| \bar{x}_{2}>=\int d \mu_{0}[\bar{x}]_{\bar{x}_{1}, \bar{x}_{2}, t} \quad e^{-\int_{0}^{t} V(\bar{x}(s)) d s}
$$

where $d \mu_{0}[\bar{x}]_{\bar{x}_{1}, \bar{x}_{2}, t}=\delta^{2}\left(\bar{x}(0)-\bar{x}_{1}\right) \delta^{2}\left(\bar{x}(t)-\bar{x}_{2}\right) d \mu_{0}[\bar{x}]$ with $d \mu_{0}[\bar{x}]$ being the Wiener measure (with the endpoints $\bar{x}(0)$ and $\bar{x}(t)$ left unspecified) of classical diffusion theory. In Euclidean time, then, the stochastic nature of quantum mechanics is indistinguishable from the classical randomness of diffusion. A quantum mechanical free particle in Euclidean time has correlation functions e.g. that are the same as those of a classical particle executing Brownian motion with diffusion constant $D=\hbar^{2} / 2 m$. A quantum particle in an external potential in Euclidean time, in its ground state say, also behaves as a diffusing classical particle, but one with the Wiener measure modified by the multiplicative factor $e^{-\int_{0}^{t} V(\bar{x}(s)) d s}$.

The free heat kernel is, in two dimensions, the familiar probability density for a particle to arrive at $\bar{x}_{2}$ at time $t$ having started at time 0 at point $\bar{x}_{1}$ :

$P_{t}^{(0)}\left(\bar{x}_{2} \mid \bar{x}_{1}\right)=h_{t}^{(0)}\left(\bar{x}_{2}, \bar{x}_{1}\right)=<\bar{x}_{2}\left|e^{-t H_{0}}\right| \bar{x}_{1}>=\int d \mu_{0}\left[\bar{x}_{\bar{x}_{1}, \bar{x}_{2}, t}=\frac{1}{4 \pi t} e^{\frac{-\left(\bar{x}_{1}-\bar{x}_{2}\right)^{2}}{4 t}}\right.$

Equivalently, for the Wiener process, the probability density function of $\bar{x}\left(t_{2}\right)-$ $\bar{x}\left(t_{1}\right)$ is:

$$
P_{\bar{x}\left(t_{2}\right)-\bar{x}\left(t_{1}\right)}(\bar{x})=\frac{1}{4 \pi\left(t_{2}-t_{1}\right)} e^{\frac{-x^{2}}{4\left(t_{2}-t_{1}\right)}}
$$

The "reproducibility property" of heat kernels:

$$
\int d^{2} y \quad h_{t_{1}}^{(0)}(\bar{x}, \bar{y}) h_{t_{2}}^{(0)}(\bar{y}, \bar{z})=h_{t_{1}+t_{2}}^{(0)}(\bar{x}, \bar{z})
$$

ensures that the conditional probability in (12) is consistent.

\section{The Prokhorov Theorem}

Implicit in the discussion above is the assumption that there exists a probability measure (the Wiener measure) on the space of continuous curves in $\mathbf{R}^{\mathbf{2}}$ which 
yields the probabilities in (12) and (13). A complete assignment of probabilities on this space of paths, however, requires that all possible events (to be defined shortly) be given probabilities consistent with the logic of probability and set theory. It is by no means obvious that the probabilities in (12) can be generalized to such a true probability measure. It is the purpose of this section to describe the Prokhorov Theorem, which provides a simple test to which the probabilities in (12) can be subjected in order to establish the existence of such a measure. The Wiener process will be seen to pass this test, and in the next section the test is used to verify that our renormalized quantum mechanical system has a path integral description in terms of a new measure which we believe to be distinct from the Wiener one in a way that systems with nonsingular potentials cannot be.

The setting that we require is a probability space, which is a triple, $(\Omega, \mathcal{B}, P)$, where $\Omega$ is a set (the sample space), $\mathcal{B}$ is a Borel algebra (an algebra of subsets of $\Omega$ closed under countable unions and complementations) whose members are the possible events, and $P: \mathcal{B} \rightarrow \mathbf{R}$ is a probability measure, meaning that it must have the properties $P(\Omega)=1$ and countable additivity, i.e.

$$
P\left(\bigcup_{n} A_{n}\right)=\sum_{n} P\left(A_{n}\right)
$$

if $A_{n} \cap A_{m}=0, \forall n \neq m$.

If instead of $\mathcal{B}$ we identify $\mathcal{A}$ as an algebra closed only under finite unions, and $p: \mathcal{A} \rightarrow \mathbf{R}$ is additive only for finite unions, then we say that $p$ is an elementary probability measure.

The probabilities given in (12) specify an elementary probability measure to the set of events of the form:

$$
E=\left\{\bar{x} \mid \bar{x} \in \Omega,\left(\bar{x}\left(t_{1}\right), \ldots, \bar{x}\left(t_{n}\right)\right) \in B^{2 n}\right\}
$$

where $B^{2 n}$ is a Borel subset of $\mathbf{R}^{\mathbf{2 n}}$ and $n$ is finite. $\mathcal{A}$ here is then the set of all such events. Their probabilities are:

$$
P(E)=\int d^{2} x_{1} \ldots \int d^{2} x_{n} P_{B^{2 n}} P_{\bar{x}\left(t_{1}\right) \ldots \bar{x}\left(t_{n}\right)}\left(\bar{x}_{1}, \ldots, \bar{x}_{n}\right)
$$

where

$$
P_{\bar{x}\left(t_{1}\right) \ldots \bar{x}\left(t_{n}\right)}=h_{t_{1}}^{(0)}\left(\overline{0}, \bar{x}_{1}\right) h_{t_{2}-t_{1}}^{(0)}\left(\bar{x}_{1}, \bar{x}_{2}\right) \ldots h_{t_{n}-t_{n-1}}^{(0)}\left(\bar{x}_{n-1}, \bar{x}_{n}\right)
$$


for $t_{1}<t_{2}<\ldots<t_{n}$. Clearly an event $E$ has many equivalent descriptions. One can always add more times to the list in (16) without placing restrictions on $\bar{x}$ at these new times without changing the event in any way. The reproducibility property of the heat kernel ensures that each of these equivalent descriptions will be assigned the same probability.

Thus with $\mathcal{A}=\{E\}$, we have defined an elementary probability measure on $\Omega$. By the Kolmogorov theorem (see e.g. [7]) if $p$ is an elementary probability measure on $\mathcal{A} \subset \Omega$ then there exists a unique extension to a full probability measure $P$ on $\mathcal{B}(\mathcal{A})$, the Borel algebra generated by $\mathcal{A}$, if and only if for any sequence of sets $A_{n} \in \mathcal{A}$ having $A_{n+1} \subset A_{n}$ and where $\bigcap_{n} A_{n}=\emptyset$, the measure has the property $\lim _{n \rightarrow \infty} p\left(A_{n}\right)=0$.

Fortunately, for our purposes, there is an equivalent, but simpler, test when $\Omega$ is the space of continuous paths. Prokhorov's theorem, [7] and [8], says that in this case the unique extension to a full probability measure exists if and only if the elementary probability measure assigns event probabilites such that, for some $t_{0}>0$, there exist constants $a>0, b>1$, and $c>0$ such that:

$$
E\left(\left|\bar{x}\left(t_{2}\right)-\bar{x}\left(t_{1}\right)\right|^{a}\right) \leq c\left|t_{2}-t_{1}\right|^{b}, \quad \forall\left|t_{2}-t_{1}\right| \leq t_{0}
$$

The Wiener process, by (13), is scale-invariant, and therefore satisfies this bound by taking $a=4$ and $b=2$.

We give an outline of the proof of Prokhorov's theorem in the Appendix. In the next section we find the heat kernel of our renormalized quantum mechanical system, and use this to define an elementary probability measure analagous to the Wiener one. Then we show that this elementary measure also can be extended to a full probability measure, thus constructing the path integral appropriate for our system.

\section{The Renormalized Path Integral}

As shown in Section 2, the renormalized delta function Hamiltonian is a self-adjoint extension of the two-dimensional Laplacian. Eigenfunctions in configuration space satisfy the free Schrodinger equation, but with a singular boundary condition at the origin. The heat kernel, then, satisfies the heat equation, with this same boundary condition at $x_{i} \rightarrow 0, i=1,2$ :

$$
-\Delta_{\bar{x}_{i}} h_{t}\left(\bar{x}_{1}, \bar{x}_{2}\right)=-\frac{\partial h_{t}\left(\bar{x}_{1}, \bar{x}_{2}\right)}{\partial t}
$$


with

$$
h_{0}\left(\bar{x}_{1}, \bar{x}_{2}\right)=\delta^{2}\left(\bar{x}_{1}-\bar{x}_{2}\right), \quad h_{t}\left(\bar{x}_{1}, \bar{x}_{2}\right) \sim C \ln \beta x_{i}, \quad x_{i} \rightarrow 0, \quad i=1,2
$$

where $\ln \left(\frac{\mu^{2}}{\beta^{2}}\right)=2(\ln 2-\gamma)$. We can get the explicit solution by first constructing the resolvent with the proper boundary conditions:

$$
G_{\lambda}\left(\bar{x}_{1}, \bar{x}_{2}\right) \equiv<\bar{x}_{1}\left|\frac{1}{-\Delta-k^{2}}\right| \bar{x}_{2}>=\frac{1}{2 \pi} K_{0}\left(k\left|\bar{x}_{1}-\bar{x}_{2}\right|\right)+\frac{1}{2 \pi} \frac{K_{0}\left(k x_{1}\right) K_{0}\left(k x_{2}\right)}{\ln \left(\frac{k}{\mu}\right)}
$$

where $\lambda=k^{2}$. The first term is the free resolvent, $G_{\lambda}^{(0)}$. The second term is required to achieve the boundary conditions for small $x_{1}$ and $x_{2}$. The resolvent is the Laplace transform of the heat kernel, so we find that the heat kernel consists of the free one plus a term which is a convolution of free heat kernels and another function, $\nu$ :

$h_{t}\left(\bar{x}_{1}, \bar{x}_{2}\right)=h_{t}^{(0)}\left(\bar{x}_{1}, \bar{x}_{2}\right)+4 \pi \mu^{2} \int_{0}^{t} d s \int_{0}^{s} d s^{\prime} h_{t-s}^{(0)}\left(\bar{x}_{1}, \overline{0}\right) \nu\left(\mu^{2}\left(s-s^{\prime}\right),-1\right) h_{s^{\prime}}^{(0)}\left(\overline{0}, \bar{x}_{2}\right)$

with $\nu$ being the function

$$
\nu(t, a) \equiv \int_{a}^{\infty} d s \frac{t^{s}}{\Gamma(s+1)}
$$

We would like to use this heat kernel to define, as in (18), an elementary probability measure on the sets in (16), and then extend this using the Prokhorov theorem to a full probability measure, thus yielding a path integral description of our renormalized Hamiltonian. The first problem we encounter is that, as when the free Hamiltonian, $H_{0}$, is modified by the addition of a potential, no longer is $\int d^{2} x_{2} h_{t}\left(\bar{x}_{2}, \bar{x}_{1}\right)=1$, so we cannot interpret $h_{t}\left(\bar{x}_{2}, \bar{x}_{1}\right)$ as a probability density (i.e. as $\left.P_{t}\left(\bar{x}_{2} \mid \bar{x}_{1}\right)\right)$. A normalization is necessary. As in the case $V \neq 0$ we can utilize the positive, normalizable, ground state $\Psi_{\lambda_{0}}(\bar{x})$ to define:

$$
P_{t}\left(\bar{x}_{2} \mid \bar{x}_{1}\right)=e^{-\mu^{2} t} \frac{\Psi_{\lambda_{0}}\left(\bar{x}_{2}\right)}{\Psi_{\lambda_{0}}\left(\bar{x}_{1}\right)} h_{t}\left(\bar{x}_{2}, \bar{x}_{1}\right)
$$

which is a normalized probability density, still has the reproducibility property, and for small times is $\sim h_{t}\left(\bar{x}_{2}, \bar{x}_{1}\right)$. In our case,

$$
\Psi_{\lambda_{0}}(\bar{x})=\frac{\mu}{\sqrt{\pi}} K_{0}(\mu x)
$$


The elementary probability measure we want is then given by (17) and (18), with $h_{t}^{(0)}$ replaced by $P_{t}$. The diffusion generated by this probability gives correlation functions and expectation values which are the quantum mechanical ground state correlation functions and expectation values in Euclidean time.

Now $P_{t}$, unlike $h_{t}^{(0)}$, is not scale-invariant, since the ground state energy $-\mu^{2}$ sets a scale. It takes, therefore, some work to show that this elementary probability measure satisfies the Prokhorov bound, and therefore has a unique extension to a probability measure on the space of continuous two-dimensional paths. The expectation value that we must bound can be written as a sum of two terms:

$$
E\left(\left|\bar{x}\left(t_{2}\right)-\bar{x}\left(t_{1}\right)\right|^{a}\right)=E^{(0)}\left(\left|\bar{x}\left(t_{2}\right)-\bar{x}\left(t_{1}\right)\right|^{a}\right)+E^{(1)}\left(\left|\bar{x}\left(t_{2}\right)-\bar{x}\left(t_{1}\right)\right|^{a}\right)
$$

where the first term corresponds to the free part of $P_{t}$, and the second term comes from the interaction part of $P_{t}$. We can bound these two postive terms separately:

$E^{(0)}\left(\left|\bar{x}\left(t_{2}\right)-\bar{x}\left(t_{1}\right)\right|^{a}\right)=\frac{\mu^{2}}{\pi} e^{-\mu^{2} t} \int d^{2} x_{1} d^{2} x_{2}\left|\bar{x}_{2}-\bar{x}_{1}\right|^{a} K_{0}\left(\mu x_{1}\right) K_{0}\left(\mu x_{2}\right) h_{t}^{(0)}\left(\bar{x}_{1}, \bar{x}_{2}\right)$

Useful here is a power expansion of $K_{0}\left(\mu x_{2}\right)$ :

$$
K_{0}\left(\mu x_{2}\right)=K_{0}\left(\mu x_{1}\right)+R\left(x_{1}, x_{2}\right)
$$

where for $\left|x_{2}-x_{1}\right|<\delta, \delta$ being small,

$$
\left|R\left(x_{1}, x_{2}\right)\right|=\left|-\mu K_{1}\left(\mu x_{1}\right)\left(x_{2}-x_{1}\right)+\ldots\right|<A(\delta) K_{1}\left(\mu x_{1}\right)\left|x_{2}-x_{1}\right|
$$

$A(\delta)$ being positive and constant with respect to $x_{1}$ and $x_{2}$. Inserting this bound on $K_{0}\left(\mu x_{2}\right)$ (note that $K_{0}$ and $K_{1}$ are positive for positive arguments) yields the bound:

$$
E^{(0)}\left(\left|\bar{x}\left(t_{2}\right)-\bar{x}\left(t_{1}\right)\right|^{a}\right)<C_{1} e^{-\mu^{2} t}\left(\mu^{2} t\right)^{a / 2}+C_{2} e^{-\mu^{2} t}\left(\mu^{2} t\right)^{\frac{a+1}{2}}
$$

where $C_{1}$ and $C_{2}$ are dimensionless constants. The other term in the expectation value we need is

$E^{(1)}\left(\left|\bar{x}\left(t_{2}\right)-\bar{x}\left(t_{1}\right)\right|^{a}\right)=\frac{\mu^{2}}{\pi} e^{-\mu^{2} t} \int d^{2} x_{1} d^{2} x_{2}\left|\bar{x}_{2}-\bar{x}_{1}\right|^{a} K_{0}\left(\mu x_{1}\right) K_{0}\left(\mu x_{2}\right) h_{t}^{(1)}\left(\bar{x}_{1}, \bar{x}_{2}\right)$ 
defining $h_{t}^{(1)}$ to be $h_{t}-h_{t}^{(0)}$, the interaction term in the heat kernel. In this expression we may use:

$$
K_{0}\left(\mu x_{1}\right) K_{0}\left(\mu x_{2}\right)<C(\epsilon)\left(x_{1} x_{2}\right)^{-\epsilon}
$$

where $\epsilon$ is any positive real number. Inserting this into the expression for $E^{(1)}$ and taking the Laplace transform with respect to $t \equiv t_{2}-t_{1}$ makes, using the resolvent formula, the integral on the right-hand side of (32)

$$
\frac{C(\epsilon)}{2 \pi \ln \frac{k}{\mu}} \int d^{2} x_{1} d^{2} x_{2}\left(x_{1} x_{2}\right)^{-\epsilon}\left|\bar{x}_{2}-\bar{x}_{1}\right|^{a} K_{0}\left(k x_{1}\right) K_{0}\left(k x_{2}\right)
$$

Conveniently, we can scale $\bar{x}_{1}$ and $\bar{x}_{2}$ to bring all the $k$ dependence outside the integral, giving us

$$
\frac{C(\epsilon, a)}{k^{4+a-\epsilon} \ln \left(\frac{k}{\mu}\right)}
$$

which has the inverse Laplace transform

$$
\frac{2 C(\epsilon, a)}{\mu^{2+a-\epsilon}} \nu\left(\mu^{2} t, \frac{a+2-\epsilon}{2}\right)
$$

The function $\nu$ has an asymptotic expansion, [9],:

$$
\nu\left(\mu^{2} t, p\right)=\frac{\left(\mu^{2} t\right)^{p}}{\ln \left(\frac{1}{\mu^{2} t}\right)}\left(C_{p}+O\left(\left|\ln \left(\frac{1}{\mu^{2} t}\right)\right|^{-1}\right)\right)
$$

Using this provides a bound on $E^{(1)}$ for small $t$ :

$$
E^{(1)}\left(\left|\bar{x}\left(t_{2}\right)-\bar{x}\left(t_{1}\right)\right|^{a}\right)<C\left(\epsilon, a, t_{0}\right) \frac{\left(\mu^{2} t\right)^{\frac{2+a-\epsilon}{2}}}{\ln \left(\frac{1}{\mu^{2} t}\right)}, \quad \forall t \leq t_{0}
$$

This result and the above bound on $E^{(0)}$ combine to prove that, as for the Wiener measure, the Prokhorov bound is satisfied with the values $a=4$ and $b=2$. This means that there is a unique probability measure, and hence path integral, to describe our renormalized quantum mechanical system. The Wiener measure in path integrals is then replaced by $d \mu_{\lambda_{0}}[\bar{x}]$ where

$$
h_{t}\left(\bar{x}_{1}, \bar{x}_{2}\right)=\int d \mu_{\lambda_{0}}[\bar{x}]_{\bar{x}_{1}, \bar{x}_{2}, t}
$$


and the addition of a potential modifies this to:

$$
h_{t}\left(\bar{x}_{1}, \bar{x}_{2}\right)=\int d \mu_{\lambda_{0}}[\bar{x}]_{\bar{x}_{1}, \bar{x}_{2}, t} \quad e^{-\int_{0}^{t} V(\bar{x}(s)) d s}
$$

Like the Wiener measure, $d \mu_{\lambda_{0}}[\bar{x}]$ is thus a measure on the space of continuous paths in $R^{2}$. An ordinary, nonsingular, interaction modifies the Wiener measure by the multiplicative factor $e^{-\int_{0}^{t} V(\bar{x}(s)) d s}$, a functional which for small times approaches unity, with corrections of $O(t)$. Under the influence of such interactions particles, for small enough times, behave as free ones. In contrast, the measure $d \mu_{\lambda_{0}}[\bar{x}]$, corresponding to the renormalized delta function interaction, also approaches the Wiener measure as $t \rightarrow 0$, but the corrections die much more slowly, being logarithmic rather than power law. This interaction, though subtle in the way it breaks scale invariance, modifies particle motion for very small times in a more profound way than does an ordinary potential. We conjecture, but have not proved, that the mathematical consequence of this is that, unlike the Wiener measure multiplied by the functional $e^{-\int_{0}^{t} V(\bar{x}(s)) d s}$, the measure $d \mu_{\lambda_{0}}[\bar{x}]$ not absolutely continuous, see e.g. [10], with respect to the free Wiener measure for arbitrarily small times.

It is also interesting to note the meaning (in the diffusion picture) of the second term in (23). This term, which is positive, corresponds to the probability that, rather than undergoing ordinary Brownian motion, the particle in going from $\bar{x}_{1}$ to $\bar{x}_{2}$, first diffuses into the neighborhood of the origin where it spends some random amount of time (the distribution of this random delay being proportional to the function $\nu$ ) before diffusing out again to its destination $\bar{x}_{2}$. In fact, keeping this picture in mind provides an alternative way to arrive at the heat kernel, $h_{t}$. Adding the probability of such an excursion to the origin amounts to adding a term $f(\lambda) G_{\lambda}^{(0)}(\bar{x}, \overline{0}) G_{\lambda}^{(0)}(\overline{0}, \bar{y})$ to the free resolvent. $f(\lambda)$ is then the Laplace transform of the random time delay distribution. The resulting ansatz for the resolvent,

$$
G_{\lambda}(\bar{x}, \bar{y})=G_{\lambda}^{(0)}(\bar{x}, \bar{y})+f(\lambda) G_{\lambda}^{(0)}(\bar{x}, \overline{0}) G_{\lambda}^{(0)}(\overline{0}, \bar{y})
$$

should then be required to have the reproducibility property, required for it to be the resolvent of some operator:

$$
\int d^{2} y G_{\lambda}(\bar{x}, \bar{y}) G_{\lambda}(\bar{y}, \bar{z})=\frac{-d}{d \lambda} G_{\lambda}(\bar{x}, \bar{z}) .
$$

Making this requirement, and using $G_{\lambda}^{(0)}(\bar{x}, \bar{y})=\frac{1}{2 \pi} K_{0}(k|\bar{x}-\bar{y}|)$, yields a firstorder nonlinear differential equation for $f$ :

$$
f^{\prime}(\lambda)=\frac{-f^{2}(\lambda)}{4 \pi \lambda}
$$


which can be integrated to give $f(\lambda)=2 \pi / \ln \left(\frac{\sqrt{\lambda}}{\mu}\right)$, where here $\mu$ appears as an integration constant. This result matches the expression given for the resolvent in (22).

Consideration then of nonrelativistic quantum particles acting through the renormalized delta function potential is equivalent to the treatment of classical particles undergoing a modified Brownian diffusion with enhanced probability for the particles to stick together for some time before going their separate ways. The possibility of such a "sticky diffusion" in two dimensions may be of interest even in classical diffusion theory or in condensed matter physics.

\section{Appendix}

Here we provide a roadmap to the proof of the Prokhorov theorem as given in [8]. The idea is to show that the "Prokhorov bound", (19), implies the conditions of the Kolmogorov theorem. That is, if the elementary probability measure implies (19), and we consider a set of events $\left\{A_{n}\right\}$ of the form (16) such that $A_{n+1} \subset A_{n}$ and $p\left(A_{n}\right)>\epsilon>0$ then $\bigcap_{n} A_{n} \neq \emptyset$.

Now, by adding times at which the particle position is unrestricted, we can always put such a sequence of events into a standard form:

$$
A_{n}=\left\{\bar{x} \mid\left(\bar{x}\left(t_{1}^{(n)}\right), \ldots, \bar{x}\left(t_{n 2^{n+1}}^{(n)}\right)\right) \in B^{n}\right\}
$$

Since $p$ is a probability measure when restricted to sets dependent on a fixed set of times, we can assume that each $B^{n}$ is a compact set. Also, by choosing $n$ large enough we can make $t_{i}^{(n)}-t_{i-1}^{(n)}<2^{-n}<t_{0}$. The Prokhorov bound and Chebyshev's inequality then give, for any $\delta>0$

$$
p\left(\bar{x}|| \bar{x}\left(t_{i}^{(n)}\right)-\left.\bar{x}\left(t_{i-1}^{(n)}\right)\right|^{a} \geq\left|t_{i}^{(n)}-t_{i-1}^{(n)}\right|^{\delta a}\right) \leq c\left|t_{i}^{(n)}-t_{i-1}^{(n)}\right|^{b-\delta a}
$$

Let $\lambda \equiv b-\delta a-1$. Using this, $p\left(A_{l}\right)>\epsilon$, and DeMorgan's law we can show that $p\left(E_{l}\right)>\frac{\epsilon}{2}$ where $\left\{E_{l}\right\}$ are the events

$$
E_{l}=A_{l} \bigcap\left(\bigcap_{n=m_{0}}^{l} \bigcap_{i=2}^{n 2^{n+1}}\left\{\bar{x}|| \bar{x}\left(t_{i}^{(n)}\right)-\bar{x}\left(t_{i-1}^{(n)}\right)|<| t_{i}^{(n)}-\left.t_{i-1}^{(n)}\right|^{\delta}\right\}\right)
$$

where $m_{0}$ is taken large enough that $2 c \sum_{n=m_{0}}^{\infty} n 2^{-n \lambda}<\frac{\epsilon}{2}$. So these sets are non-empty. The event $E_{l}$ includes all paths which belong to $A_{l}$ and in addition have the property that in each time division between $m_{0}$ and $l$, i.e. for $m_{0}<n<l$, the distance travelled is bounded above by $\Delta t^{\delta}$. 
Now it is clear that $E_{l+1} \subset E_{l} \subset A_{l}$ so that showing $\bigcap_{l=m_{0}}^{\infty} E_{l} \neq \emptyset$ is sufficient to prove $\bigcap_{l=m_{0}}^{\infty} A_{l} \neq \emptyset$. To show the former, first pick from each set $E_{l}$ a path, $\bar{x}_{l}(t)$, which is linear in each time segment, $\left[t_{i-1}^{(l)}, t_{i}^{(l)}\right]$. Geometry and the definition of $E_{l}$ imply that for $t_{i}^{(l)} \leq t \leq s \leq t_{j}^{(l)}$

$$
\left|\bar{x}_{l}(t)-\bar{x}_{l}(s)\right| \leq K\left|t_{i}^{(l)}-t_{j}^{(l)}\right|^{\delta}
$$

for a constant $K>0$. Now the paths $\bar{x}_{l+p}$ belong to $A_{l}$ for all $p>0$, and therefore $\left(\bar{x}_{l+p}\left(t_{1}^{(l)}\right), \ldots, \bar{x}_{l+p}\left(t_{l 2^{l+1}}^{(l)}\right)\right) \in B^{l} . \quad B^{l}$ is compact. Therefore this sequence, indexed by $p$, has a limit point in $B^{l}$. This is true for all $l$. Thus we can by the diagonalization method extract a subsequence, $\left\{\bar{y}_{n}\right\}$ such that $\bar{y}_{n}\left(t_{i}^{(l)}\right)$ converges as $n \rightarrow \infty$ for all $i$ and $l$. Now, if $\eta$ and $\tau$ are given, we can choose $n_{0}$ large enough such that $t_{i}^{\left(n_{0}\right)} \leq \tau \leq t_{i+1}^{\left(n_{0}\right)}$ and $\left|t_{i}^{\left(n_{0}\right)}-t_{i+1}^{\left(n_{0}\right)}\right|<2^{-n_{0}}<\frac{\eta}{2}$. Then choosing $l$ and $m$ large enough, we can show by triangle inequalities that $\left|\bar{y}_{l}(\tau)-\bar{y}_{m}(\tau)\right|<A \frac{\eta}{2}$ for some positive constant $A$. This is true for any $\tau \in\left[t_{i}^{\left(n_{0}\right)}, t_{i+1}^{\left(n_{0}\right)}\right]$. Thus the limiting function, say $\bar{y}(t)$, exists $\forall t \in \mathbf{R}$, and (47) ensures that $\bar{y}(t)$ is continuous. $\bar{y}(t)$ has the property $\left(\bar{y}\left(t_{1}^{(l)}\right), \ldots \bar{y}\left(t_{l 2^{l+1}}^{(l)}\right)\right) \in B^{l}, \forall l$, so that $\bar{y} \in \bigcap_{l \geq m_{0}} E_{l} \neq \emptyset$, completing the proof.

\section{References}

1. C. Thorn, Phys. Rev. D19, 639 (1979).

2. K. Huang, "Quarks, Leptons and Gauge Fields", World Scientific, Singapore (1982).

3. K.S. Gupta and S.G. Rajeev, Phys. Rev. D48, 5940 (1993).

4. C. Manuel and R. Tarrach, Phys. Lett. B328, 113 (1994).

5. S. Albeverio, F. Gesztesy, R. Hoegh-Krohn, and H. Holden, "Solvable Models in Quantum Mechanics", Springer-Verlag, New York, NY (1988)

6. R.J. Henderson and S.G. Rajeev, Intl. J. Mod. Phys. A 10, 3765 (1995).

7. Y.V. Prokhorov, Theory of Prob. and its Applic. 1, 157 (1956).

8. "Lectures on Stochastic Processes", by K. Ito, notes by M.Rao, TIFR (1961)

9. Erdelyi, A. (Editor), The Bateman Manuscript Project, Higher Transcendental Functions, Vol III, p217, McGraw-Hill, New York, NY (1955)

10. D. Freedman, "Brownian Motion and Diffusion", Holden-Day, Inc., San Francisco, CA (1971) 